\title{
Conocimientos y prácticas de profesionales de la salud en uso de medicamentos
}

\author{
Knowledge and practices of health professionals in the use of medicines \\ Conhecimentos e práticas de profissionais da saúde no uso de medicamentos
}

María Zoraida Rojas-Marín *

\begin{abstract}
Resumen
Identificar conocimientos y prácticas del personal de salud respecto al uso de medicamentos en vías y condiciones diferentes a las establecidas en la ficha técnica. Método: Estudio cuantitativo de tipo descriptivo y corte transversal en dos fases: la primera consistió en una revisión de la literatura en donde se incluyeron de forma inicial 82 artículos, de los cuales ocho fueron elegidos para la revisión; la segunda fase estuvo conformada por 31 participantes que trabajaban en Instituciones de Salud; fueron seleccionados de forma no probabilista secuencial, a quienes se les aplicó un cuestionario, para identificar conocimientos y prácticas desarrolladas, el cual fue evaluado por 3 profesores expertos en la temática; también se realizó una prueba piloto. Resultados: La revisión de la literatura fue referente para la construcción del instrumento. En la segunda fase el $87 \%$ de los participantes no conocían el concepto off label, el $65 \%$ fueron profesionales de la enfermería que describieron su experiencia como una práctica que no se podría considerar como off label por la falta de soporte científico. Los medicamentos antiinfecciosos, antifúngicos, analgésicos y electrolitos, fueron los grupos de mayor utilización. La principal modificación fue en el cambio de la vía de administración, seguido de la indicación y el $71 \%$ de las prácticas no fue descrita en los registros clínicos. Conclusiones: Existe limitada evidencia que soporte el uso de medicamentos en vías y condiciones no establecidos en la ficha técnica; no existe una definición clara y común, presentándose un desconocimiento en los profesionales de la salud.
\end{abstract}

Palabas Clave: Uso fuera de lo indicado, tratamiento farmacológico, preparaciones farmacéuticas, utilización de medicamentos
Autor de correspondencia

* Enfermera. Magister en CienciasFarmacología. Especialista en Docencia Universitaria y Enfermería Cardiorrespiratoria. Docente-investigadora. Profesora asistente Universidad El Bosque. Correo: rojasmmariaz@gmail.com. Orcid: https://orcid.org/0000-0002-1228-9214. Bogotá, Colombia.

\begin{abstract}
Identify the knowledge and practices of the health personnel regarding the use of medicine different from the procedures and conditions established in the medical fact sheet. Method: Quantitative study of descriptive and cross-sectional type: the first consisted of a review of the literature where 82 articles were initially included, from which eight were selected for review; the second one was made by 31 participants who worked at healthcare institutions; these were selected by sequential sampling, and a questionnaire was used to identify the knowledge and practices developed, the
\end{abstract}

(C) Universidad Francisco de Paula Santander. Este es un artículo bajo la licencia CC BY (https://creativecommons.org/ licenses/by/4.0/) @) (1) 
ISSN-PRINT

1794-9831

E-ISSN 2322-7028

Vol. 17 No. 1

Ene - Abr 2020

Cúcuta, Colombia

questionnaire was evaluated by 3 expert professors; a pilot experiment was also made. Results: The review of the literature was a reference to build the instrument. On the second phase, $87 \%$ of the participants did not know the concept off label, and $65 \%$ were nursing professionals who described their experience as a practice that cannot be considered off label due to the lack of scientific support. The anti-infective, anti-fungal, analgesics and electrolytes were the most used drugs. The main adjustment was the administration procedure, followed by the indications, and $71 \%$ of the practices were not described in the case report form. Conclusions: There is limited evidence that supports the use of medicine different from the procedures and conditions that are not established in the medical fact sheet; there is no clear and common definition, implicating unknowingness of the healthcare professionals.

Keywords: Use different from the indications, pharmacological treatment, pharmaceutical preparations, use of medicine.

\section{Resumo}

Identificar conhecimentos e práticas do pessoal de saúde respeito ao uso de medicamentos em vías e condições diferentes às estabelecidas na bula. Método: Estudo quantitativo, descritivo de corte transversal realizado em duas fases: a primeira, realizou-se uma revisão da literatura onde incluíram-se inicialmente 82 artigos, dos quais 8 elegeram-se para a revisão; a segunda fase, estudou 31 participantes trabalhadores do setor saúde selecionados de maneira não probabilística sequencial aplicando-se um questionário para estabelecer os conhecimentos e práticas desenvolvidas, avaliado previamente por 3 professores expertos na temática; também foi realizada uma prova piloto. Resultados: A revisão da literatura esteve relacionada com a construção do instrumento. Na segunda fase o 87\% dos participantes não conhecia o conceito off label pela ausência de suporte científico. Os medicamentos antibacterianos, antifúngicos, analgésicos e eletrólitos foram os de maior utilização. A principal modificação foi a mudança na via de administração, seguido da indicação e o $71 \%$ das práticas não foram registradas no prontuário. Conclusões: Existe limitada evidencia que suporte o uso de medicamentos nas vias e condições não estabelecidas na bula; não existe definição clara e comum, presentando-se o desconhecimento nos profissionais da saúde.

Palavras-chave: Prescrições de medicamentos, tratamento farmacológico, preparações farmacêuticas, uso de medicamentos.

\section{Introducción}

El aumento de los medicamentos como una de las medidas más recurrentes e importantes en el proceso de recuperación y mantenimiento de la salud de la población, demandan una continua actualización de los conocimientos sobre los fármacos y del uso racional de los mismos, en todos los profesionales de las ciencias de la salud. Los medicamentos indicados, seleccionados y administrados erróneamente pueden tener efectos drásticos, produciendo serios perjuicios o daños al paciente. Estos errores pueden mitigarse al seguir las instrucciones dadas en los resúmenes científicos sobre las características del medicamento relacionadas con diferentes aspectos: indicaciones, posología, contraindicaciones, efectos adversos, precauciones para su empleo, condiciones de conservación, etc; ésta información científica es esencial para que los profesionales de la salud tomen las mejores decisiones. Este documento se conoce como la ficha técnica o la IPP (información para prescribir). Este es un tema de interés y actualización para los profesionales de la salud y especialmente para los de enfermería, en razón a que gran parte del ejercicio profesional del cuidado directo de los pacientes se basa en la interpretación, administración y evaluación de la respuesta de los tratamientos farmacológicos.

La administración de medicamentos tiene un proceso (prescripción, transcripción, preparación y administración), y al realizar este proceso con alguna modificación, se pueden alterar o disminuir las características fisicoquímicas del medicamento lo que puede comprometer el efecto o presentar eventos adversos (1). El enfermero/a en este proceso tiene un compromiso legal, pero sobre todo ético, para actuar con responsabilidad frente a las acciones, indicaciones y contraindicaciones de estas sustancias, por lo que el conocimiento de la Farmacología dentro de este colectivo es fundamental (2); no obstante, se pueden presentar prácticas inusuales del personal de enfermería, ya sean por una acción individual o por 
indicación del personal médico, que pueden modificar las condiciones de los fármacos desde la indicación, forma farmacéutica del medicamento o vía, conocido esto como usos Off label.

El uso de medicamentos fuera de las indicaciones aprobadas o el uso de Off label, es una práctica en discusión frente a la cual no existe una posición clara por parte de los profesionales de la salud, las industrias farmacéuticas o las entidades reguladoras (3). El término Off-Label o fuera de la indicación, no es muy conocido; sin embargo, el hecho de no conocer su concepto, no implica que necesariamente no se realicen este tipo de prácticas en los contextos hospitalarios. Es un tema frente al cual no existe unanimidad entre las diferentes agencias regulatorias, y muchas de ellas incluso no tienen una posición clara frente a este tema. La Food and Drug Administration (FDA), por ejemplo, define el empleo de la expresión "off label de medicamentos" como el uso de un medicamento para una indicación, forma farmacéutica, régimen de dosificación, población o cualquier otro parámetro no mencionado en la etiqueta que ha sido aprobada por la entidad regulatoria. Simultáneamente en la literatura médica anglosajona estas prácticas se describen como "unlicensed" cuando el medicamento se utiliza en prácticas no autorizadas y "off-label" cuando se lo usa en condiciones distintas a las autorizadas (4).

El uso de medicamentos fuera de la ficha técnica puede contemplar tres características diferentes (5):

- Uso del Fármaco con una indicación totalmente distinta a la aprobada en la ficha técnica.

- Uso del Fármaco con la misma indicación aprobada, pero en distintos subgrupos de pacientes (pacientes pediátricos o mujeres embarazadas, por ejemplo).

- Uso del Fármaco en condiciones distintas a las aprobadas, por ejemplo, a mayor o menor dosis, una duración de tratamiento distinta, o por una vía de administración diferente de la autorizada.

A su vez, en Europa como un esfuerzo en la unificación de criterios frente al uso de off label de medicamentos y al uso no licenciado, se establece la siguiente terminología, la cual es sugerida a la European Medicines Agency $\operatorname{EMA}(6)$ :

- Uso off-label: Uso para un medicamento comercializado que no esté en el SPC (Summary of Pro-
ductCharacteristics) incluyendo indicación terapéutica, uso en subgrupos etarios, dosificación, forma farmacéutica y ruta de administración.

- Uso off-label pediátrico: Usos pediátricos para un medicamento comercializado que no esté en el SPC, en población pediátrica ( 0 a 18 años de edad).

- Uso no licenciado: Todos los usos para un medicamento que nunca ha recibido una European Marketing Authorization para uso humano tanto en adultos como en niños

Adicionalmente, se debe contemplar el concepto de ficha técnica o Summary of Product Characteristics (SPC), como aquel documento legal y de soporte científico que va destinado a los profesionales de la salud, en el que se incluye un resumen de información crucial sobre un medicamento como las indicaciones terapéuticas, posología y modo de administración, contraindicaciones, efectos adversos, precauciones en el uso, interacciones con otros medicamentos y condiciones de conservación, entre otros aspectos. "El laboratorio que comercializa el medicamento solamente garantiza la calidad, la eficacia y la seguridad de éste en las indicaciones y las condiciones de uso que figuran en la ficha técnica"(7). Callejas (8) refiere que la ficha técnica "no es un documento estático, ya que las agencias reguladoras pueden autorizar la modificación del contenido dependiendo de la aparición de nuevas evidencias sobre el medicamento: datos de seguridad a largo plazo, ensayos clínicos para nuevas indicaciones terapéuticas, cambios en los ajustes posológicos"(8). Lo planteado anteriormente, indica la importancia de este documento para el profesional de la salud en el ámbito laboral, ya que proporciona una guía para el uso del medicamento, evitando y disminuyendo efectos adversos para el paciente y brindando una mayor seguridad al tratamiento.

En Colombia el Instituto de Vigilancia de Medicamentos y Alimentos (INVIMA), la agencia regulatoria de este país, la considera aceptable cuando existe evidencia que la respalde y, así, permite la incorporación y el uso de medicamentos vitales en circunstancias particulares. Por otra parte, se puede hacer un uso diferente al autorizado oficialmente por el INVIMA, y es aceptable si está sustentado en estudios clínicos adecuadamente realizados; sin embargo, no hay una regulación clara frente a este tema y se desconoce la magnitud del problema en el país. (9) 
ISSN-PRINT

1794-9831

E-ISSN 2322-7028

Vol. 17 No. 1

Ene - Abr 2020

Cúcuta, Colombia
Dentro de las diferentes posiciones frente a este tema, se ha planteado que el uso de medicamentos fuera de las indicaciones aprobadas, puede estar justificado en aquellos casos donde requiera una alternativa terapéutica; no obstante, como desventaja de esta práctica se destaca el desconocimiento de los efectos adversos, lo cual puede exponer al paciente a no alcanzar el objetivo terapéutico (6). Para la inclusión de un fármaco de uso off-Label es necesaria la consideración de puntos importantes como: evidencia científica que soporte su uso y atención oportuna desde la política de seguridad del paciente, ya que este uso puede generar reacciones adversas por desconocimiento; de igual forma, si el medicamento es de alto costo, su uso debe probar su efectividad a la hora de la obtención de resultados clínicos (10).

El uso de medicamentos en vías y condiciones diferentes a las establecidas en su ficha técnica, es un tema en creciente auge, continuándose con un amplio desconocimiento por parte de los profesionales de la salud, quienes realizan acciones de modificación, en función de sus creencias individuales o por prácticas direccionadas. Estos procedimientos frecuentemente no tienen soportes de evidencias fuertes sobre la vía y modificación de las formas farmacéuticas; también, se reconoce que son prácticas que no justifican su realización, considerándose como errores de medicación. Teniendo en cuenta lo planteado anteriormente, se puede decir que, cuando se prescribe y dispensa fuera de lo concretamente especificado en cuanto al empleo de off label, ni la eficacia ni la seguridad de este uso pueden garantizarse.

Por los motivos antes descritos, el uso de medicamentos bajo las condiciones de modificación, exige que se siga investigando para aportar nuevas evidencias. Es por eso, que en este caso que nos compete, como profesionales de la enfermería, debemos tener intervenciones más razonadas y con la suficiente evidencia para su realización; igualmente, hay que disminuir los problemas relacionados con los medicamentos y fortalecer su administración, asegurando que su eficacia, seguridad y calidad se mantengan en todas las fases del proceso.

\section{Objetivos}

\section{Objetivo General}

Identificar los conocimientos y prácticas del personal de salud respecto al uso de medicamentos en condiciones y vías de administración no incluidas en su etiqueta o ficha técnica

\section{Objetivo Específicos}

- Identificar la evidencia científica existente para soportar conocimientos, prácticas y creencias respecto a la modificación de las condiciones para el uso de medicamentos.

- Describir las prácticas de profesionales de la salud entorno al uso de medicamentos, clasificando las prácticas en uso off-label o posibles errores en la preparación y/o administración de medicamentos.

\section{Materiales y Métodos}

Se realizó una investigación cuantitativa descriptiva de corte transversal, la cual se desarrolló en 2 fases, durante los meses de enero 2014 a noviembre 2016.

\section{Primera Fase}

Consistió en la elaboración de una revisión de la literatura con la estandarización de criterios de inclusión, dentro de los cuales se estableció la ventana de tiempo, correspondiente a los periodos comprendidos entre los años 2000 y 2014. Se realizó una búsqueda exhaustiva en diferentes bases de datos. Se aplicaron los criterios de los idiomas: español, portugués e inglés.

Las bases de datos consultadas fueron: Proquest, Pubmed, NursingConsult, Medline, Lilacs, ScienceDirect, Cochrane. Para la búsqueda de información se establecieron palabras claves mediante el uso de DECS y MESH, utilizados en el área de la salud. En el proceso inicial de la revisión de conocimientos y prácticas de profesionales de la salud, acerca del uso de medicamentos en vías y condiciones no incluidas en su ficha técnica, se realizó la búsqueda general de los artículos mediante el uso de los siguientes descriptores: "off- Label", conocimientos, ficha técnica, licencia, unlicensed; obteniendo un total de 82 artículos, los cuales al efectuar el filtro final con la lectura del documento completo, permitió seleccionar finalmente un total de 8 artículos para el análisis.

\section{Segunda Fase}

Se identificaron las prácticas del personal de Enfermería frente a las modificaciones de la indicación, forma farmacéutica y vía de administración de medicamentos. Se tomó una muestra no probabilística de manera secuencial por conveniencia, para una población de 500 participantes, con una proporción esperada del $87 \%$, una precisión del $12 \%$ y una confiabilidad del $95 \%$; también, se calculó un tamaño de muestra de 29 personas, en donde se in- 
cluyeron 31 participantes, quienes se encontraban laborando en servicios de hospitalización de 6 Instituciones de Salud de II, III y IV nivel de atención, en la ciudad de Bogotá. Se elaboró un formulario con 19 preguntas tipo Likert, el cual fue evaluado por 3 profesores expertos en la temática, y posterior aplicación de la prueba piloto. Las variables de interés correspondieron al conocimiento sobre el concepto de medicamentos off label y las prácticas de modificación de la vía, indicación y forma farmacéutica de los medicamentos. La recolección de los datos la realizaron 7 estudiantes que participaron como asistentes de investigación.

Los datos fueron digitados en una matriz elaborada en Excel versión 2013 y procesados con la ayuda de estadísticas descriptivas como frecuencias absolutas, porcentajes, desviaciones estándar, valores máximos y mínimos y coeficientes de variación.

Respecto a los aspectos éticos de la investigación, se consideró el estudio sin riesgo, de acuerdo con lo establecido en la Resolución 008430 del 4 de octubre de 1993, emitida por el Ministerio de Salud colombiano. La decisión de los participantes fue voluntaria y podían retirarse en cualquier momento si así lo deseaban. Se administró un consentimiento informado, con manejo confidencial.
Se contó con el aval del Comité de Investigación de la Facultad de Enfermería de una Institución de educación superior, Acta 132 del 27 de febrero de 2014.

\section{Resultados}

\section{Fase 1: Revisión de la literatura}

Se realizó una revisión sobre literatura relacionada con artículos que trataran temas que tuvieran que ver con conocimientos y prácticas de profesionales de la salud, respecto al uso de medicamentos en vías y condiciones diferentes a las establecidas en la ficha técnica. Se utilizaron los siguientes criterios de búsqueda: Off - Label; conocimientos, actitudes y prácticas en salud; ficha técnica y etiqueta; uso fuera de lo indicado en la etiqueta. Se excluyeron artículos que hacían referencia a la prescripción y evaluación de fármacos. De los documentos analizados, la mayor población correspondió a un $66 \%$ en profesionales de la medicina, un $22 \%$ en profesionales de la Enfermería y un $11 \%$ de varios profesionales de la salud. En la tabla 1 se presenta el resumen de la revisión de la literatura.

Tabla 1. Resultados sobre el uso de medicamentos en la práctica

\begin{tabular}{|c|c|c|c|c|c|}
\hline Autor/año & Titulo & Objetivo & Población & Hallazgos significativos & Conclusiones \\
\hline $\begin{array}{l}\text { García-Sa- } \\
\text { bina, Et al. } \\
\text { Farm Hosp } \\
2011(10)\end{array}$ & $\begin{array}{l}\text { Revisión sobre } \\
\text { el uso de me- } \\
\text { dicamentos en } \\
\text { condiciones no } \\
\text { incluidas en su } \\
\text { ficha técnica }\end{array}$ & $\begin{array}{l}\text { Obtener relación } \\
\text { de fármacos con } \\
\text { usos off-label en } \\
\text { enfermedades fre- } \\
\text { cuentes y ofrecer } \\
\text { lista como refe- } \\
\text { rencia. }\end{array}$ & $\begin{array}{l}2 \text { manuales } \\
\text { de atención, } \\
13 \text { guías } \\
\text { clínicas y } 14 \\
\text { normas de } \\
\text { actuación de } \\
\text { urgencias }\end{array}$ & $\begin{array}{l}\text { De } 310 \text { enfermedades, se obtuvieron } 69 \\
(22,3 \%) \text { con descripción del uso de fárma- } \\
\text { cos off-label. Detallan información sobre } \\
\text { usos off-label de fármacos analizado: in- } \\
\text { dicación, mecanismo de acción, tratamien- } \\
\text { to bajo esquema terapéutico, aprobación } \\
\text { FDA, dosis y demás aspectos que se consi- } \\
\text { deren peculiares ante uso off-label. }\end{array}$ & $\begin{array}{l}\text { Se recomienda disponer de una } \\
\text { lista como referencia sobre las } \\
\text { enfermedades en las que fre- } \\
\text { cuentemente se utilizan medica- } \\
\text { mentos off-label. } \\
\text { Propone guías y protocolos que } \\
\text { permitan realizar efectivamente } \\
\text { la validación de prescripciones. }\end{array}$ \\
\hline $\begin{array}{l}\text { Gimenes } \\
\text { Fernanda R. } \\
\text { et al. Rev. } \\
\text { Latino-Am. } \\
\text { Enferma- } \\
\text { gem } 2011 \\
\text { (11) }\end{array}$ & $\begin{array}{l}\text { Administración } \\
\text { de medica- } \\
\text { mentos en vías } \\
\text { diferentes de } \\
\text { las indicadas en } \\
\text { la prescripción } \\
\text { médica }\end{array}$ & $\begin{array}{l}\text { Analizar la in- } \\
\text { fluencia de la } \\
\text { redacción de la } \\
\text { prescripción mé- } \\
\text { dica en la admi- } \\
\text { nistración de me- } \\
\text { dicamentos }\end{array}$ & $\begin{array}{l}1.425 \text { situa- } \\
\text { ciones de } 5 \\
\text { hospitales } \\
\text { brasileños }\end{array}$ & $\begin{array}{l}92 \text { situaciones donde el medicamento ad- } \\
\text { ministrado estaba en discordancia con la } \\
\text { prescripción médica. Casos en los cuales } \\
\text { la administración de medicamentos era por } \\
\text { vías que no estaban aprobadas, resaltando } \\
\text { la administración por sonda nasogástrica } \\
\text { de medicamentos prescritos por vía oral. }\end{array}$ & $\begin{array}{l}\text { La mayoría de las prescripcio- } \\
\text { nes fueron electrónicas, conte- } \\
\text { nían siglas y o abreviaturas tales } \\
\text { como SC (subcutánea) y GTT } \\
\text { (gastrostomía), lo que podría } \\
\text { conllevar al error. }\end{array}$ \\
\hline $\begin{array}{l}\text { De Bortoli- } \\
\text { ni. et al. et } \\
\text { al. Cienc. } \\
\text { enferm. } 2010 \\
\text { (12) }\end{array}$ & $\begin{array}{l}\text { Identificación } \\
\text { y análisis de } \\
\text { los errores de } \\
\text { medicación en } \\
\text { seis hospitales } \\
\text { brasileños }\end{array}$ & $\begin{array}{l}\text { Este estudio iden- } \\
\text { tificó los errores } \\
\text { de medicación } \\
\text { ocurridos en uni- } \\
\text { dades de clínica } \\
\text { médica de } 6 \text { hos- } \\
\text { pitales brasileños }\end{array}$ & $\begin{array}{l}\mathrm{n}=35 \text { dosis/ } \\
\text { día Seis } \\
\text { hospitales en } \\
\text { total. }\end{array}$ & $\begin{array}{l}\text { El error puede ocurrir en cualquier momen- } \\
\text { to, ya que el proceso de medicación ocurre } \\
\text { en varias etapas secuenciales, dependien- } \\
\text { tes una de otra y ejecutadas por un equipo } \\
\text { multidisciplinar compuesto por médicos, } \\
\text { farmacéuticos, enfermeras/ros y auxiliares }\end{array}$ & $\begin{array}{l}\text { En este estudio se evidencio que } \\
\text { el } 1.5 \% \text { de los medicamentos } \\
\text { fueron administrados en vías di- } \\
\text { ferentes a las establecidas en su } \\
\text { ficha técnica indicando que no } \\
\text { se pueden clasificar como usos } \\
\text { off label y si en error de admi- } \\
\text { nistración que puede ocasionar } \\
\text { efectos adversos en el paciente. }\end{array}$ \\
\hline
\end{tabular}


ISSN-PRINT

1794-9831

E-ISSN 2322-7028

Vol. 17 No. 1

Ene - Abr 2020

Cúcuta, Colombia
Neubert el al. Pharmacological Research 2008 (13)

Belén RuízAntorán et al. Journal of Pediatric Gastroenterology and Nutrition. 2013(14)

\begin{tabular}{|c|c|}
\hline $\begin{array}{l}\text { Chalu- } \\
\text { meau. et al. } \\
\text { Archives of } \\
\text { Disease in } \\
\text { Childhood } \\
2000(15)\end{array}$ & $\begin{array}{l}\text { Off label and } \\
\text { unlicensed drug } \\
\text { use among } \\
\text { French office } \\
\text { based pediatri- } \\
\text { cians }\end{array}$ \\
\hline
\end{tabular}

2000 (15) cians
Drug Utilization and Off-label Drug Use in Spanish Pediatric Gastroenterology Outpatients
Describir y cuantificar los medicamentos recibidos por los niños atendidos en el departamento de gastroenterología pediátrica, su uso fuera de etiqueta, y el cumplimiento de las normas aceptadas para dicho uso. 2522 presización de me- cripciones dicamentos fuera de medicade la etiqueta y mentos a 989 el consumo de pacientes. medicamentos sin licencia.

695 pacientes menores de 2 años.

2522 pres-
cripciones
de medica-
mentos a 989
pacientes.


Saullo et al. A questionnai2013 (17) re-based study in Calabria on the knowledge of off-label drugs in pediatrics
Evaluar el cono- Pediatras cimiento de me- miembros de dicamentos off la sociedad label en pediatras italiana de de la región de pediatras, Calabria, Italia. pediatras.

Los pediatras prescribieron en total de 341 medicamentos off label adjuntos en 154 categorías de enfermedades. $40 \%$ de los pediatras refirió que "algunas veces" usaban medicamentos off label. El uso más común fue el de la edad.

66 pediatras usan medicamentos off label por falta de formulaciones aprobadas para uso pediátrico.
ISSN-PRINT

1794-9831

E-ISSN 2322-7028

Vol. 17 No. 1

Ene - Abr 2020

Cúcuta, Colombia

\section{Fuente: Autora}

Con la revisión de la literatura, se identificó que existe una limitada evidencia que soporte los conocimientos y usos de medicamentos en vías y condiciones no establecidos en la ficha técnica; por tanto, no existe una definición clara y se presenta un desconocimiento en los profesionales de la salud $(10,13-15,17)$. Otras características comunes sobre los resultados de las investigaciones son: el uso fuera de etiqueta de forma común entre los pediatras, dado que existe falta de medicamentos específicamente diseñados y comercializados para niños de diferentes grupos de edad (13-17); información ausente o reducida para la correcta utilización de los medicamen- tos; alto riesgo de errores y eventos relacionados con los medicamentos $(11,12,14,17)$.

\section{Resultados Fase 2}

Se aplicó el instrumento a 31 participantes, donde se evidencia una mayor participación de mujeres (81\%), siendo esta una característica constante en la disciplina de la enfermería. El rango de edad fue de 21 a 56 años. El $65 \%$ de la población eran profesionales en enfermería, de los cuales el 40\% (9 enfermeras/os) había realizado algún estudio adicional de posgrado. Los datos se muestran en la tabla 2.

Tabla 2. Características sociodemográficas

\begin{tabular}{lll}
\hline Sexo & $\mathrm{n}=31$ & $\%$ \\
\hline Hombre & 6 & $19 \%$ \\
Mujer & 25 & $81 \%$ \\
\hline Nivel de escolaridad & $\mathrm{n}=31$ & $\%$ \\
\hline Técnico & 11 & $35 \%$ \\
Profesional & 20 & $65 \%$ \\
Pregrado & 11 & $55 \%$ \\
Especialización & 8 & $40 \%$ \\
Maestría & 1 & $5 \%$ \\
Tiempo de ejercicio profesional & $\mathrm{n}=31$ & $\%$ \\
\hline 7 años & 15 & $48 \%$ \\
$>7$ años & 16 & $52 \%$ \\
Institución donde se desarrolla actualmente su actividad & $\mathrm{n}=31$ & $\%$ \\
2 Nivel (1) & 4 & $13 \%$ \\
3 Nivel (2) & 13 & $42 \%$ \\
4 Nivel (3) & 12 & $39 \%$ \\
\hline
\end{tabular}

Fuente: Autora

\section{Conocimientos y prácticas de uso de medicamentos fuera de las condiciones}

Con el autodiligenciamiento del cuestionario, se obtuvieron respuestas sobre los usos de medicamentos desde el propio ejercicio o del conocimiento de la práctica de otro profesional, la cual en el estudio se denomina como ajena. Se pueden presentar limitaciones de información en este componente, ya que al ser una práctica ajena se desconocen algunos datos importantes. La tabla 3, presenta el consolidado de la información. 
ISSN-PRINT

1794-9831

E-ISSN 2322-7028

Vol. 17 No. 1

Ene - Abr 2020

Cúcuta, Colombia

Tabla 3. Conocimientos y prácticas de uso de medicamentos fuera de las condiciones

\begin{tabular}{|c|c|c|c|}
\hline Categoría & Variable & $\mathrm{N}=31$ & $\%$ \\
\hline \multirow[t]{2}{*}{ Conocimiento concepto medicamentos off label } & $\mathrm{Si}$ & 4 & 13 \\
\hline & No & 27 & 87 \\
\hline \multirow[t]{2}{*}{ Realización de la modificación del medicamento } & Actividad realizada por el mismo profesional-auxiliar & 21 & 67 \\
\hline & Actividad realizada por otro profesional-auxiliar & 10 & 33 \\
\hline \multirow[t]{2}{*}{ Actividad considerada por ellos mismos como práctica off label } & $\mathrm{Si}$ & 11 & 35 \\
\hline & No & 20 & 65 \\
\hline \multirow[t]{3}{*}{ Persona que indica la realización de la práctica } & Médico & 18 & 58 \\
\hline & Colega & 9 & 29 \\
\hline & Otro & 4 & 13 \\
\hline \multirow[t]{3}{*}{$\begin{array}{l}\text { Tiempo de ejercicio profesional en el momento de realizar la práctica de } \\
\text { modificación del medicamento }\end{array}$} & Menor a 5 años & 16 & 52 \\
\hline & mayor a 5 años & 11 & 35 \\
\hline & No sabe & 4 & 13 \\
\hline \multirow[t]{4}{*}{ Práctica de modificación de la condición del medicamento } & Vía & 19 & 61 \\
\hline & Indicación & 4 & 13 \\
\hline & Forma farmacéutica & 4 & 13 \\
\hline & Vía e indicación & 4 & 13 \\
\hline \multirow[t]{4}{*}{ Información que respaldó la práctica } & Otros profesionales & 12 & 38 \\
\hline & Protocolo o guía & 6 & 19 \\
\hline & Revista científica & 1 & 3 \\
\hline & Ninguna & 13 & 41 \\
\hline \multirow[t]{7}{*}{$\begin{array}{l}\text { Diagnósticos de los pacientes a los que se les realizó la modificación del } \\
\text { medicamento CIE } 10\end{array}$} & Migraña no especificada & 4 & 13 \\
\hline & Trastorno de ansiedad generalizada & 3 & 10 \\
\hline & Trastornos del metabolismo del potasio & 3 & 10 \\
\hline & Traumatismo & 2 & 6 \\
\hline & Aborto espontáneo & 2 & 6 \\
\hline & Gastritis no especificada & 2 & 6 \\
\hline & Otros & 15 & 48 \\
\hline \multirow[t]{4}{*}{ Grupo de medicamentos que presentaron la modificación } & antibióticos y antifúngicos & 8 & 26 \\
\hline & Analgésicos & 6 & 19 \\
\hline & Electrolitos & 5 & 16 \\
\hline & Otros (sedantes, antiulcerosos, inductores,) & 11 & 35 \\
\hline \multirow[t]{3}{*}{ Desenlace en cumplimiento del fin terapéutico } & $\mathrm{Si}$ & 24 & 76 \\
\hline & No & 5 & 17 \\
\hline & No sabe & 2 & 7 \\
\hline \multirow[t]{3}{*}{ Presencia de efectos indeseados } & $\mathrm{Si}$ & 2 & 7 \\
\hline & No & 24 & 77 \\
\hline & No sabe & 5 & 16 \\
\hline \multirow[t]{2}{*}{$\begin{array}{l}\text { Realiza el registro especificando la modificación en el uso del medica- } \\
\text { mento }\end{array}$} & $\mathrm{Si}$ & 9 & 29 \\
\hline & No & 22 & 71 \\
\hline
\end{tabular}

\section{Fuente: Autora}




\section{Discusión}

Los resultados de la revisión de la literatura, presentan de forma prioritaria las modificaciones que tienen que ver con las indicaciones en condiciones distintas a las aprobadas. Los resultados referentes al cambio de vía y modificaciones de la forma farmacéutica son más limitados. Estos resultados permiten evidenciar la existencia de múltiple literatura de profesionales en Medicina, como lo refiere Randall (18) quien menciona que el uso que se le da a los medicamentos de manera diferente a la aprobada por la FDA (Administración de Alimentos y Medicamentos) es legal y muy común por parte de los médicos, por la simple razón de que no existe una evidencia bien definida que soporte este tipo de prácticas. Adicionalmente Saullo et al. (17) exponen que este uso de medicamentos es muy común entre los pediatras, y la causa principal se debe a la falta de medicamentos específicamente diseñados y comercializados para niños de diferentes grupos de edad.

Aun cuando esta temática tiene un interés e incremento creciente, todavía existe un amplio desconocimiento del concepto y regulación como lo mencionan Cordoba (3) y Buitrago et al. (23) cuando indican que cerca del $73 \%$ de los medicamentos usados fuera de las indicaciones aprobadas, no tienen un fundamento o respaldo científico que avale su uso, lo cual puede exponer al paciente a un riesgo de seguridad para una pobre respuesta terapéutica (19, 20); sin embargo Ghinea et al, (21) en contraposición, en su estudio concluyen que la percepción de médicos australianos es que las etiquetas no son guías significativas para la práctica clínica y que existen mecanismos alternativos para garantizar la prescripción segura, racional y basada en la evidencia.

La revisión de la literatura desarrollada en la primera fase permitió determinar que el uso de medicamentos off label se da principalmente en el personal médico, quien en la mayoría de los países, incluido Colombia, es quien prescribe.

Adicionalmente, la limitada información de las modificaciones en los usos, vías y formas farmacéuticas, principalmente por el personal de la Enfermería, plantea la necesidad de aportar al área de conocimiento, desarrollando la segunda fase del presente estudio. En ésta se identificó como primera causa la modificación de la vía, seguida de la indicación (22); situación contraria al estudio de Chalumeau et al.(15) quienes señalan que la primera causa fue la indicación y finalmente la modificación de la forma farmacéutica, que de igual manera se relacionarían con el cambio de la vía. Esto difiere de unos de los resultados de la revisión de la literatura de la primera fase, ya que se observa una influencia en relación con la indicación original del producto farmacéutico.

Entre el grupo de medicamentos que presentaron mayores modificaciones están: los antibióticos, los antifúngicos, los analgésicos y los electrolitos. Las prácticas de modificación se relacionan con la modificación de la vía, principalmente en el uso de ampollas por vía oral, como en el caso del midazolam, ranitidina, potasio ampolla estéril diluida en jugo de naranja, dipirona diluida en café o directa. Algunas de estas prácticas tienen como soporte la literatura relacionada con el uso off label como es el caso de la dipirona (23) pero no diluida en bebidas, y el midazolam (24) específicamente en población pediátrica. En este aparte se debe considerar que la variación de las formas farmacéuticas y las vías en condiciones diferentes a las de los estudios de elaboración, sus excipientes y las condiciones de fabricación, son muy importantes en relación con la liberación del principio activo en la luz del tubo digestivo y también en lo que se refiere a la velocidad de absorción en el organismo. Depende de la vía respecto a los factores fisiológicos como el $\mathrm{pH}$, la solubilidad del medicamento; estos factores pueden tener una serie de limitaciones por el $\mathrm{pH}$ ácido y las enzimas proteolíticas, alterando el efecto terapéutico y generando problemas de destrucción de revestimientos, pudiendo irritar la mucosa gástrica (25).

Por su parte los hallazgos referidos a la modificación de las formas farmacéuticas como es el caso de: los polvos liofilizados como la ampicilina sulbactam para ser administrado de forma tópica, el liofilizado de vancomicina para ser administrado vía oral, la modificación de las cápsulas de diversos antifúngicos y antibióticos para ser utilizadas de forma tópica, no tienen soporte para ser consideradas como usos off label, por el contrario, se podrían considerar como errores de medicación de vía.

Las experiencias de las prácticas referidas por los participantes del estudio respecto al desenlace positivo de la modificación del medicamento, el cual estaba orientado al cumplimiento del objetivo terapéutico para lo cual fue indicado, obtuvo un efecto terapéutico y seguro sin reportar presencia de reacciones adversas. Este hallazgo está en concordancia con lo que refieren García et al.(26) quienes exponen que el uso de medicamentos off-Label han tenido efectos terapéuticos deseados tras utilizar este mecanismo; sin embargo, 
ISSN-PRINT

1794-9831

E-ISSN 2322-7028

Vol. 17 No. 1

Ene - Abr 2020

Cúcuta, Colombia el presente estudio no tuvo la incorporación de otras variables de seguimiento para verificar que la práctica (que no siempre fue off label) por sí misma diera el efecto terapéutico deseado. Por esta misma razón, la ausencia de reacciones adversas no se da como definitiva; Aya y Suárez (27) refieren que estas prácticas pueden traer consigo una serie de consecuencias negativas, las cuales se pueden prevenir. Por su parte Mckean y Monasterio (28) exponen que el uso de medicamentos fuera de las indicaciones establecidas, cuya evidencia científica no es sólida, tiene una gran correlación con la aparición de reacciones adversas a los medicamentos RAM y se ha demostrado que estas prescripciones son un factor de riesgo para el desarrollo de futuras reacciones adversas a medicamentos, errores en medicación y la posibilidad de fallo terapéutico (29).

Tanto en la revisión de la literatura, como en los resultados de la segunda fase, la ausencia de registros de las prácticas es evidente. Esto puede conllevar a riesgos mayores, ya que, desde los fundamentos legales, toda acción del personal de salud debe proporcionar información, consentimiento y registro en la historia clínica (30); y más aún, cuando es una intervención que no se encuentra bien definida en el ejercicio práctico.

\section{Conclusiones}

En los últimos años se presenta un incremento en la información e investigaciones respecto a los conocimientos y usos off label principalmente en la indicación por parte del personal médico. Sin embargo, en lo que tiene que ver con los conocimientos y prácticas en la modificación de vías y condición (forma farmacéutica, dosis) la evidencia es limitada y casi ausente.

Los participantes presentan desconocimiento del concepto de medicamento off label. No existe una definición comúnmente aceptada y hay desconocimiento por parte del personal de salud, respecto a las generalidades e implicaciones del uso de los medicamentos en condiciones no aprobadas.

Las principales prácticas de modificación se encuentran en el cambio de la vía, seguido de la modificación de las formas farmacéuticas.

Los participantes de la presente investigación refieren que la modificación realizada no estaba soportada con evidencia científica para identificarla claramente como un uso Off-label. Por lo tanto, se puede clasificar como un error en la administración de los medicamentos.

Es necesario continuar realizando investigaciones que presenten la problemática que se puede generar; adicionalmente, se deben fundamentar las prácticas y uso de medicamentos en vías y condiciones no establecidas en la ficha técnica, identificando la causa, el problema y las consecuencias, para así generar guías e información suficiente que permitan tomar decisiones razonadas y orientar el uso seguro de medicamentos.

\section{Agradecimientos.}

La autora agradece a Stephania Avilés, Catalina Cárdenas, Sandra Milena Cortes, Claudia Andrea Gutiérrez, Marta Yannet Pico, Karen Rincón y Victoria Roa, estudiantes de la Facultad de Enfermería de la Universidad el Bosque, por su apoyo en la recolección de los datos y sus comentarios sobre la propuesta inicial.

\section{Conflicto De Intereses}

La autora declara no tener ningún conflicto de intereses.

\section{Referencias Bibliográficas}

1. Hernández Pérez MA, Mestre Zarate MB, Mora Rodríguez L, Ramírez Suarez NE. Factores asociados a errores en la administración de medicamentos por el profesional de enfermería en una institución de tercer nivel en Cartagena, 2013. [Trabajo de grado]. Cartagena de Indias. Corporación Universitaria Rafael Núñez; 2013.

2. Ospina A, Benjumea D, Amariles PL. Problemas de proceso y resultado relacionados con los medicamentos: evolución histórica de sus definiciones. RevFac. Nac. [Internet]. 2011 [Consultado 07 de Octubre de 2015]; 29(3):329-340. Disponible en: http://www.scielo.org.co/scielo.php?pid=S0120386X2011000300014\&script $=$ sci abstract $\&$ tlng $=$ es 
3. Córdoba O. Determinación de la prescripción y uso de medicamentos no POS, con indicación no aprobada, en los servicios pediátricos dentro de una IPS de cuarto nivel de complejidad, Bogotá D.C. 2015. [Tesis]. Bogotá. Pontificia Universidad Javeriana;2017.

4. Cortessi M. Uso "off label" y contraindicado de medicamentos: cuestiones éticas y responsabilidad profesional. Blog Farma y salud. [Internet]. 2013 [Consultado 18 de febrero 2018]. Disponible en: http://farmaysalud.blogspot.com/2013/04/uso-off-label-y-contraindicado-de.html

5. Blanco Reina E. Disponibilidad de medicamentos en situaciones especiales: uso compasivo y usos offlabel. ActFarmTerap. [Internet]. 2012 [Consultado 20 de noviembre de 2017]; 10(1):43-49. Disponible en: https://dialnet.unirioja.es/servlet/articulo?codigo $=3997850$

6. Fletscher P. Uso de psicofármacos fuera de las indicaciones aprobadas en una entidad promotora de salud de Bogotá 2012. [Tesis]. Bogotá. Universidad nacional de Colombia;2012.

7. Instituto para el Uso Seguro de Medicamentos ISMP. ¿Qué es un error de medicación? [Internet]. 2015 [Consultado el 03 de Abril de 2017]. Disponible en: http://www.ismp-espana.org/estaticos/view/19

8. Callejas Ibáñez P. Reconocimiento de enfermedades en fichas técnicas de medicamentos y su anotación con snomed-ct. [Tesis de Maestría] Madrid:Universidad Politécnica de Madrid; 2015. [Consultado el 29 de octubre de 2019]. Disponible en: http://oa.upm.es/35518/1/CALLEJA_IBA\%C3\%91EZ_PABLO_2015.pdf

9. Vanegas E, Acosta A, Vaca C, López J, Pineda L, Guzmán J, et al. Propuesta de un modelo de gestión de medicamentos en indicaciones no registradas en Colombia. RevPanam Salud Publica. [Internet]. 2016 [Consultado 16 de junio de 2018]; 39(5):274-280. Disponible en: http://iris.paho.org/xmlui/bitstream/handle/123456789/28524/v39n5a11_274-280.pdf?sequence $=1$

10. García A, Rabunal R, Martínez R. Revisión sobre el uso de medicamentos en condiciones no incluidas en su ficha técnica. Farmacia Hospitalaria [Internet]. 2010 [Consultado 1 de marzo 2016];35(5):264277. Disponible en: http://www.sefh.es/fh/118 121v35n05pdf009.pdf

11. Gimenes F, Escobar R, Marques T, Thalyta T, Cardoso A,Sabóia M, et al. Administración de medicamentos en vías diferentes de las indicadas en la prescripción médica. Rev. Latino-Am. Enfermagem [Internet].2011 [consultado 26 de junio 2018]; 19(1):11-17. Disponible en: http://dx.doi.org/10.1590/ $\underline{\text { S0104-11692011000100003 }}$

12. De BortoliCassiani S, MonzaniAline S, Bauer De Camargo S,Trevisani F, Perufo S, Cardoso T.Identificación y análisis de los errores de medicación en seis hospitales brasileños. Cienc. enferm. [Internet]. 2010 [consultado 2018 Jun 26]; 16(1):85-95. Disponible en: http://dx.doi.org/10.4067/ $\underline{\mathrm{S} 0717-95532010000100010}$

13. Neubert A, Wong I, Bonifazi A, Catapano M, Felisi M, et al. Defining off-label and unlicensed use of medicines for children: Results of a Delphi survey. PharmacologicalResearch [Internet]. 2008 [consultado 20 de junio de 2016]; 58(6):316-322. Disponible en: https://doi.org/10.1016/j.phrs.2008.09.007

14. RuízAntorán B,Piñeiro R, Avendaño C, Román E, Cilleruelo M, Gutiérrez C, et al. Drug Utilization and Off-label Drug Use in Spanish Pediatric Gastroenterology Outpatients.Journal of Pediatric Gastroenterology and Nutrition. [Internet]. 2013 [consultado 2 junio 2017] 56(2):173-7. Disponible en : https://www.ncbi.nlm.nih.gov/pubmed/23328455

15. Chalumeau M, Tréluyer JM, Salanave B, Assathiany R, Cheron G, Crocheton N, et al. Off label and unlicensed drug use among French office based pediatricians. Archives of Disease in Childhood [Internet]. 2002 [consultado 18 marzo 2018]; 83(6):502-505.Disponible en; https://www.ncbi.nlm.nih. gov/pmc/articles/PMC1718582/

16. Carvalho C,Ribeiro M, Bonilha M, FernandesJr M, Procianoy R, Silveira R. Uso de medicamentos off-label e não licenciados emunidade de tratamento intensivo neonatal e suaassociaçãocom escores de gravidade. J. Pediatr. (Rio J.) [Internet]. 2012 [consultado 20 junio 2017];88(6):465-470 Disponible en: http://www.scielo.br/scielo.php?script=sci arttext\&pid=S0021-75572012000600004 
ISSN-PRINT

$1794-9831$

E-ISSN 2322-7028

Vol. 17 No. 1

Ene - Abr 2020

Cúcuta, Colombia

17. Saullo F, Saullo E, Caloiero M, Menniti M, Carbone C, Chimirri S, et al. A questionnaire-based study in Calabria on the knowledge of off-label drugs in pediatrics. Journal of Pharmacology \&Pharmacotherapeutics [Internet]. 2013 [consultado 24 de junio] 4(5):51-54. Disponible en: http://doi. org/10.4103/0976-500X.120960

18. Randall S. Regulating Off-Label Drug Use - Rethinking the Role of the FDA. N Engl J Med[Internet]. 2008 [consultado 24 de junio 2019]; 358(14):1427-9.Disponible en: https://www.ncbi.nlm.nih.gov/ pubmed/18385495

19. Memorias IV encuentro Nacional. INVIMA. Calderón Herrera. Perspectiva del uso de medicamentos fuera de registro. Papel del Médico. Instituto de evaluación tecnológica en salud. 2012. Disponible en: $\quad$ https://www.invima.gov.co/images/pdf/farmacovigilancia alertas/eventos/IV-ENCUENTRONACIONAL/MEMORIAS/1-10-Perspectiva-del-uso-de-medicamentos-off-label-en-el-mundo-y-elpapel-del-medico-en-Colombia.pdf

20. Martínez C, Bravo J. Prescripción off-label inducida: Responsabilidad ¿compartida?.RevPediatr Aten Primaria [Internet]. 2016 Mar [citado 20 junio 2018]; 18(69)59-63. Disponible en: http://scielo.isciii. es/scielo.php?script $=$ sci_arttext\&pid=S1139-76322016000100016\&lng=es.

21. Ghinea N, Kerridge,Little, Lipworth .Challenges to the validity of using medicine labels to categorize clinical behavior: An empirical and normative critique of "offlabel" prescribing. Evaluación in cliniclpractice. [Internet]. 2016 [consultado 20 junio 2018]; 23(3). Disponible en:https://onlinelibrary.wiley. com/doi/pdf/10.1111/jep. 12673

22. NirNeuman H, Abu Kishk I, Toledano M, Heyman E, ZivBaran T, Berkovitch M. Unlicensed and Off-Label Medication Use in Pediatric and Neonatal Intensive Care Units: No Change Over a Decade. AdvTher. [Internet]. 2018 [consultado 15 junio 2018]; 35(7):1122-32. Disponible en:https://doi. org/10.1007/s12325-018-0732-y

23. Buitrago González T, Calderón Ospina C, Vallejos Narváez A. Dipirona: ¿Beneficios subestimados o riesgos sobredimensionados? Revisión de la literature. Rev. colomb. cienc. quim. farm. [Internet]. 2014 [consultado 20 June 2018]; 43(1):173-195. Disponible en:http://dx.doi.org/10.15446/rcciquifa. $\mathrm{v} 43 \mathrm{n} 1.45472$

24. Gómez L, Ocampo F, Orozco J, Caicedo J. Eficacia de la premedicación anestésica en el paciente pediátrico con midazolam oral y acetaminofén. Estudio observacional. Rev. colombanestesiol. [Internet]. 2013 [Consultado Marzo 17 de 2017];41(1):4-9. Disponible en: http://www.scielo.org.co/pdf/rca/ v41n1/v41n1a02.pdf

25. Red Iris. Formas farmacéuticas y vías de administración de fármacos. Universidad Autónoma de Madrid, Departamento de Farmacología y Terapéutica. Facultad de Medicina. [Internet]. 2010 [Consultado 31 de marzo 2017].Disponible en: http://bscw.rediris.es/pub/bscw.cgi/d829269/Formas\%20 farmac $\% \mathrm{C} 3 \% \mathrm{~A} 9$ uticas $\% 20 \mathrm{y} \% 20 \mathrm{v} \% \mathrm{C} 3 \% \mathrm{ADas} \% 20 \mathrm{de} \% 20 \mathrm{administraci} \% \mathrm{C} 3 \% \mathrm{~B} 3 \mathrm{n} \% 20 \mathrm{de} \% 20$ f\%C3\%A1rmacos.pdf

26. García A, Rabunal R, Martínez. Revisión sobre el uso de medicamentos en condiciones no incluidas en su ficha técnica. Farmacia Hospitalaria. [Internet]. 2010 [Consultado 17 de febrero 2017]; 35(5):264277. Disponible en: http://www.sefh.es/fh/118_121v35n05pdf009.pdf

27. Aya A, Suárez A. Percepción de los factores relacionados con el error en la administración de medicamentos en el servicio de hospitalización de una institución colombiana de cuarto nivel. Rev.Col.Enf. [Internet]. 2014 [Consultado 17 de febrero 2016]; 9(2):21-30. Disponible en:https://doi.org/10.18270/ rce.v9i9.561

28. Mckean A, Monasterio E. Off-Label Use of Atypical Antipsychotics Cause for Concern. Springer. CNSDrugs. [Internet]. 2012 [Consultado 29 octubre de 2017]; 26(5):383-390. Disponible en: https:// link.springer.com/article/10.2165\%2F11632030-000000000-00000

29. Diez G. Dosificación "off label" en paciente pediátrico. Estudio transversal en un hospital terciario. 
Universidad autónoma de Madrid. [Tesis Internet]. 2016 [Consultado junio 23 de 2018].Disponible en:https://repositorio.uam.es/handle/10486/680453

30. Piñeiro R, Ruiz MB, Román E. Conocimiento sobre el uso de fármacos off-Label en Pediatría. Resultados de una encuesta pediátrica nacional 2012-2013 (estudio OL-PED). AnPediatr. [Internet]. 2014 [Consultado febrero 26 de 2017]; 81(1):16-21. Disponible en: http://www.analesdepediatria.org/es/ conocimiento-sobre-el-uso-farmacos/articulo/S1695403313004086/

31. Cortessi M. Uso "off label" y contraindicado de medicamentos: cuestiones éticas y responsabilidad profesional. Blog Farma y salud. [Internet]. 2013 [Consultado 18 de febrero 2018]. Disponible en: http://farmaysalud.blogspot.com/2013/04/uso-off-label-y-contraindicado-de.html

32. Blanco Reina E. Disponibilidad de medicamentos en situaciones especiales: uso compasivo y usos off-label. Act Farm Terap. [Internet]. 2012 [Consultado 20 de noviembre de 2017]; 10(1):43-49. Disponible en: https://dialnet.unirioja.es/servlet/articulo?codigo $=3997850$

33. Fletscher P. Uso de psicofármacos fuera de las indicaciones aprobadas en una entidad promotora de salud de Bogotá 2012. [Tesis]. Bogotá. Universidad nacional de Colombia; 2012.

34. Instituto para el Uso Seguro de Medicamentos ISMP. ¿Qué es un error de medicación? [Internet]. 2015 [Consultado el 03 de Abril de 2017]. Disponible en: http://www.ismp-espana.org/estaticos/view/19

35. Callejas Ibáñez P. Reconocimiento de enfermedades en fichas técnicas de medicamentos y su anotación con snomed-ct. [Tesis de Maestría] Madrid: Universidad Politécnica de Madrid; 2015. [Consultado el 29 de octubre de 2019]. Disponible en: http://oa.upm.es/35518/1/CALLEJA_IBA\%C3\%91EZ_PABLO_2015.pdf

36. Vanegas E, Acosta A, Vaca C, López J, Pineda L, Guzmán J, et al. Propuesta de un modelo de gestión de medicamentos en indicaciones no registradas en Colombia. Rev Panam Salud Publica. [Internet]. 2016 [Consultado 16 de junio de 2018]; 39(5):274-280. Disponible en: http://iris.paho.org/xmlui/bitstream/handle/123456789/28524/v39n5a11_274-280.pdf?sequence $=1$

37. García A, Rabunal R, Martínez R. Revisión sobre el uso de medicamentos en condiciones no incluidas en su ficha técnica. Farmacia Hospitalaria [Internet]. 2010 [Consultado 1 de marzo 2016]; 35(5):264277. Disponible en: http://www.sefh.es/fh/118 121v35n05pdf009.pdf

38. Randall S. Regulating Off-Label Drug Use - Rethinking the Role of the FDA. N Engl J Med [Internet]. 2008 [consultado 24 de junio 2019]; 358(14):1427-9. Disponible en: https://www.ncbi.nlm.nih. gov/pubmed/18385495

39. Memorias IV encuentro Nacional. INVIMA. Calderón Herrera. Perspectiva del uso de medicamentos fuera de registro. Papel del Médico. Instituto de evaluación tecnológica en salud. 2012. Disponible en: https://www.invima.gov.co/images/pdf/farmacovigilancia alertas/eventos/IV-ENCUENTRONACIONAL/MEMORIAS/1-10-Perspectiva-del-uso-de-medicamentos-off-label-en-el-mundo-y-elpapel-del-medico-en-Colombia.pdf

40. Martínez C, Bravo J. Prescripción off-label inducida: Responsabilidad ¿compartida?. Rev Pediatr Aten Primaria [Internet]. 2016 Mar [citado 20 junio 2018]; 18(69)59-63. Disponible en: http://scielo.isciii. es/scielo.php?script=sci_arttext\&pid=S1139-76322016000100016\&lng=es.

41. Ghinea N, Kerridge,Little, Lipworth .Challenges to the validity of using medicine labels to categorize clinical behavior: An empirical and normative critique of "off $\square$ label" prescribing. Evaluación in clinicl practice. [Internet]. 2016 [consultado 20 junio 2018]; 23(3). Disponible en: https://onlinelibrary. wiley.com/doi/pdf/10.1111/jep.12673

42. Nir Neuman H, Abu Kishk I, Toledano M, Heyman E, Ziv Baran T, Berkovitch M. Unlicensed and Off-Label Medication Use in Pediatric and Neonatal Intensive Care Units: No Change Over a Decade. Adv Ther. [Internet]. 2018 [consultado 15 junio 2018]; 35(7):1122-32. Disponible en: https://doi. org/10.1007/s12325-018-0732-y

43. Buitrago González T, Calderón Ospina C, Vallejos Narváez A. Dipirona: ¿Beneficios subestimados $\overline{\text { E-ISSN 2322-7028 }}$

Vol. 17 No. 1

Ene - Abr 2020

Cúcuta, Colombia 
ISSN-PRINT

$1794-9831$

E-ISSN 2322-7028

Vol. 17 No. 1

Ene - Abr 2020

Cúcuta, Colombia o riesgos sobredimensionados? Revisión de la literature. Rev. colomb. cienc. quim. farm. [Internet]. 2014 [consultado 20 June 2018]; 43(1):173-195. Disponible en: http://dx.doi.org/10.15446/rcciquifa. v43n1.45472

44. Gómez L, Ocampo F, Orozco J, Caicedo J. Eficacia de la premedicación anestésica en el paciente pediátrico con midazolam oral y acetaminofén. Estudio observacional. Rev. colomb anestesiol. [Internet]. 2013 [Consultado Marzo 17 de 2017]; 41(1):4-9. Disponible en: http://www.scielo.org.co/pdf/ rca/v41n $1 / \mathrm{v} 41 \mathrm{n} 1 \mathrm{a} 02 . \mathrm{pdf}$

45. Red Iris. Formas farmacéuticas y vías de administración de fármacos. Universidad Autónoma de Madrid, Departamento de Farmacología y Terapéutica. Facultad de Medicina. [Internet]. 2010 [Consultado 31 de marzo 2017]. Disponible en: http://bscw.rediris.es/pub/bscw.cgi/d829269/Formas\%20 farmac $\%$ C3 $\%$ A 9 uticas $\% 20 \mathrm{y} \% 20 \mathrm{v} \% \mathrm{C} 3 \% \mathrm{ADas} \% 20 \mathrm{de} \% 20 \mathrm{administraci} \% \mathrm{C} 3 \% \mathrm{~B} 3 \mathrm{n} \% 20 \mathrm{de} \% 20$ f\%C3\%A1rmacos.pdf

46. García A, Rabunal R, Martínez. Revisión sobre el uso de medicamentos en condiciones no incluidas en su ficha técnica. Farmacia Hospitalaria. [Internet]. 2010 [Consultado 17 de febrero 2017]; 35(5):264277. Disponible en: http://www.sefh.es/fh/118_121v35n05pdf009.pdf

47. Aya A, Suárez A. Percepción de los factores relacionados con el error en la administración de medicamentos en el servicio de hospitalización de una institución colombiana de cuarto nivel. Rev.Col.Enf. [Internet]. 2014 [Consultado 17 de febrero 2016]; 9(2):21-30. Disponible en: https://doi.org/10.18270/ rce.v9i9.561

48. Mckean A, Monasterio E. Off-Label Use of Atypical Antipsychotics Cause for Concern. Springer.CNS Drugs. [Internet]. 2012 [Consultado 29 octubre de 2017]; 26(5):383-390. Disponible en: https://link. springer.com/article/10.2165\%2F $11632030-000000000-00000$

49. Diez G. Dosificación "off label" en paciente pediátrico. Estudio transversal en un hospital terciario. Universidad autónoma de Madrid. [Tesis Internet]. 2016 [Consultado junio 23 de 2018]. Disponible en: https://repositorio.uam.es/handle/10486/680453

50. Piñeiro R, Ruiz MB, Román E. Conocimiento sobre el uso de fármacos off-Label en Pediatría. Resultados de una encuesta pediátrica nacional 2012-2013 (estudio OL-PED). An Pediatr. [Internet]. 2014 [Consultado febrero 26 de 2017]; 81(1):16-21. Disponible en: http://www.analesdepediatria.org/es/ conocimiento-sobre-el-uso-farmacos/articulo/S1695403313004086/ 